\title{
Medial part thickness of wearable device affecting running motion
}

\author{
Naoki Kubota ${ }^{1 *}$ (I) and Yasuhisa Hasegawa ${ }^{2}$
}

\begin{abstract}
This paper reports negative effects on runner's motions which are supported by an exoskeleton attached to his/her lower limbs. A wearable assistive device has generally been developed to support motions of a wearer, while the device may disturb wearer's motions due to physical features, including expanded his/her body outline and device's weight. The expanded outline can exert adverse influence that limits range of motion of a wearer. In this paper, we focus on lower limb motions of a runner who puts an exoskeleton on his/her lower limbs to receive physical assistance. We simulated influence of medial parts of the exoskeleton on running through experiments with seven runners wearing one of three couples of rectangular-shaped light blocks (10 mm, $20 \mathrm{~mm}$ or $30 \mathrm{~mm}$ in thickness) on the medial sides of both thighs. These blocks increased step width but the $30 \mathrm{~mm}$-thick blocks only increased net running heat rates by $6.05 \mathrm{bpm}$ compared with that of running without blocks. This result shows that a wearable device which is equipped with components less than $20 \mathrm{~mm}$-thick on each medial part of a runner's thigh has little detrimental effects on running motions.
\end{abstract}

Keywords: Running assistance, Exoskeleton for running, Step width

\section{Introduction}

Researchers have developed devices augmenting and supporting healthy human motions including lifting up, hopping and walking [1]. A target motion to be augmented has shifted from static motions to dynamic motions. Grabowski and Herr [2] developed an exoskeleton for supporting human hopping. This exoskeleton attached parallel springs to wearer's legs and supported leg motions. It reduced net metabolic power by $24 \%$ compared with that during hopping without the exoskeleton. For walking assistance, Kitatani et al. [3] developed a powered device which supported hip joint extension and flexion. They measured oxygen consumption during walking at comfortable speed with or without the device. This assistive device was reported to reduce net oxygen consumption by $7.06 \%$ during walking at comfortable speed compared with that without the device. Collins et al. [4] developed an unpowered exoskeleton assisting

\footnotetext{
*Correspondence: kubota.naoki@d.mbox.nagoya-u.ac.jp

${ }^{1}$ The Department of Micro-Nano Systems Engineering, Nagoya University, Furo-cho 1, Chikusa-ku, Nagoya, Aichi 464-8603, Japan Full list of author information is available at the end of the article
}

walking. This exoskeleton supported plantar flexion of the ankle joints with springs, and reduced net metabolic power during walking by $7.2 \pm 2.6 \%$ compared with that without it. Luke et al. [5, 6] developed a powered walking assistance device supporting plantar flexion of the ankle joints. They reported that their device reduced net metabolic cost during walking by $11 \pm 4 \%$ compared with that without it. Kim et al. [7, 8] have developed a running assistance device supporting hip joint extension. This device powered to drive actuators reduced net metabolic power during running by $3.9 \%$ and $8.2 \%$ compared with that without the device and that with the unpowered device, respectively. They reported negative effects of this device, increasing metabolic power with un-actuated device from that without the device.

The purpose of these assistive devices is to reduce human effort for motions. Eventual effects of the devices are summation of positive effects and negative effects of the devices. To increase the eventual effects, increase in positive effects and decrease in negative effects are required. This paper focuses on reducing the negative effects on running to increase the eventual effects. Mass and its distribution of the device are important for in the 
negative effects' reduction. Bourdin et al. [9] measured the effects on running at $5 \mathrm{~m} / \mathrm{s}$ by weight jackets, called vertical loading. They reported that the jacket which was $10 \%$ weight of body weight increased net energy cost by $4.6 \%$ compared with that without the jacket. Martin [10] evaluated the effects by additional weights on thighs, shanks and feet. This research reported that $1.0 \mathrm{~kg}$ weights attached to each foot and thigh increased oxygen consumption of runners by $7.2 \%$ and $3.5 \%$ compared with that without any weights, respectively. The oxygen consumption with load was reported to linearly increase according to mass between 0.0 and $1.0 \mathrm{~kg}$ on feet and thighs. This research showed relationship between metabolic demand and two properties of objects attached to the legs: weights and positions.

Since effects of additional weight on a runner have been reported, designers of assistive devices can estimate negative effects of device's weight. On the other hand, effects of volume of the device on a runner have not reported. Range of motion of a person who wears an exoskeleton on his/her body may be limited because his/her expanded body tends to collide with each other. This limitation becomes severer at a close area between body parts, such as knee and ankle. For example, medial parts of a wearable device attached to the legs are expected to increase step width, distance on the mediolateral axis between right and left foot landing points, during running due to reduced margin between the legs. The medial parts are essential for structure design of attachments and medial joints of the devices around knee and ankle joints. Walking and running with wider step width than normal one need higher metabolic power $[11,12]$. In this paper, we measure motions and heart rates during running experiments with blocks attached to medial sides of the thighs. We propose design requirements about the shape of wearable devices supporting running motions to reduce negative effects of the devices. These devices are supported to be used by healthy adult people who are commuting and delivering mails for lower running energy cost.

\section{Experimental setup Subjects}

Seven healthy male runners served as subjects for this study. Table 1 shows the subject measurements, and Fig. 1 shows definition of the variables. The leg length (LL) is distance between a maker on the most lateral prominences of the grater trochanter (GT) and the ground in an upright position. The thigh length is distance between the GT and a marker on the lateral epicondyle of the femur. Block position is distance between the GT and the center of the blocks on the proximodistal axis of the legs. Knee width is distance
Table 1 Subject measurements (mean $\pm s d, n=7$ )

\begin{tabular}{ll}
\hline Variable & Value \\
\hline Age & $23 \pm 1.6$ years \\
Height & $1.70 \pm 0.05 \mathrm{~m}$ \\
Weight & $61.59 \pm 7.72 \mathrm{~kg}$ \\
Leg length & $0.85 \pm 0.03 \mathrm{~m}$ \\
Thigh length & $0.37 \pm 0.02 \mathrm{~m}$ \\
Block position & $0.30 \pm 0.03 \mathrm{~m}$ \\
Knee width & $0.12 \pm 0.02 \mathrm{~m}$ \\
\hline
\end{tabular}

between the most lateral prominence of the lateral femoral epicondyle and the medial prominence of the medial femoral epicondyle. All the subjects were rightlegged runners. Informed consent was obtained from all subjects prior to their participation in the study. The experiments of this study were approved by the ethics committee of Nagoya University.

\section{Test procedures}

Four conditions were employed to examine effects by medial parts attached to the legs. A subject ran wearing one of following objects on each thigh: (1) no block, (2) $10 \mathrm{~mm}$-thick blocks, (3) $20 \mathrm{~mm}$-thick blocks and (4) $30 \mathrm{~mm}$-thick blocks. Figure 2a shows the blocks. The subjects putted the blocks on medial sides of the thighs using tapes, and these tapes were slack enough for muscle contraction (Figs. 1, 2b). The location of the blocks

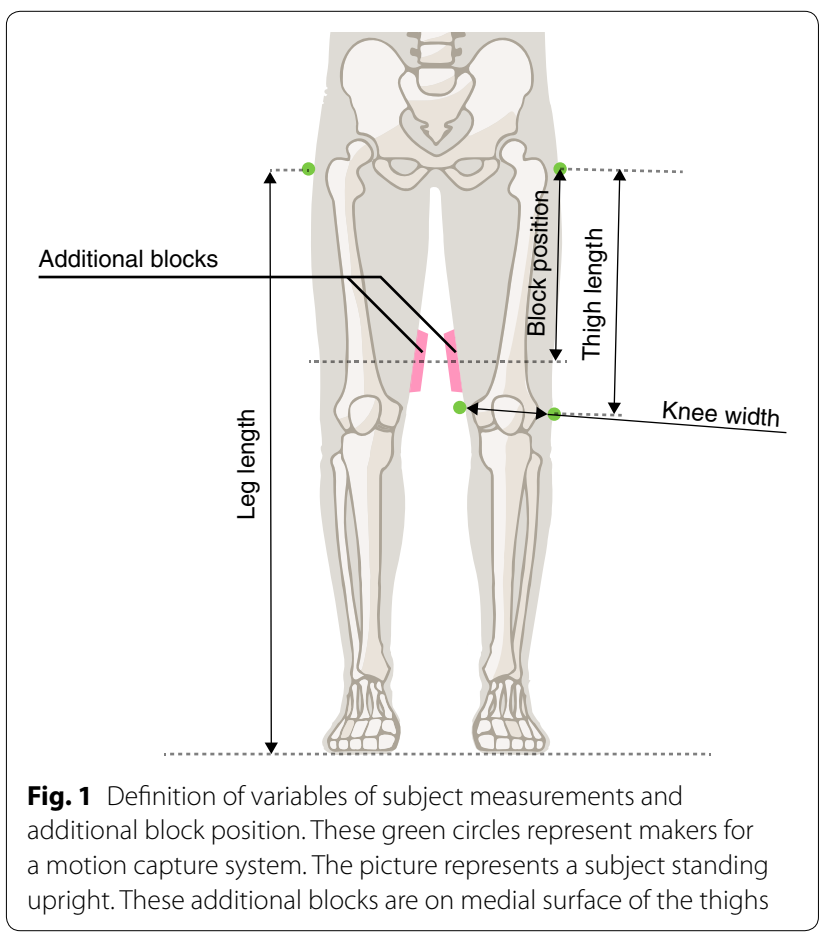




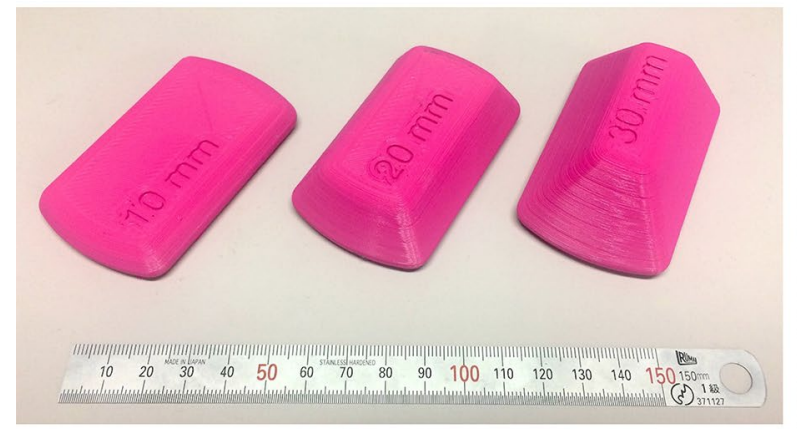

a

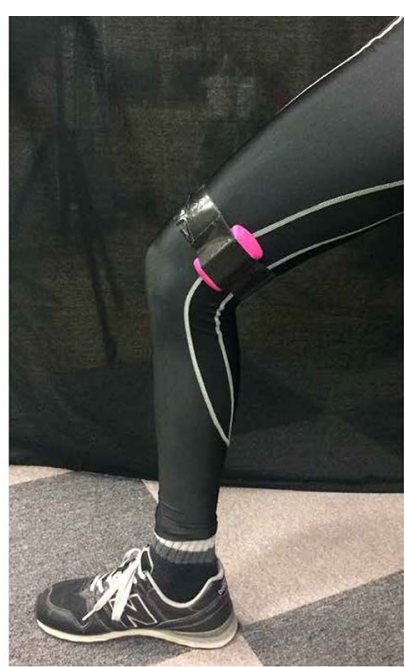

b

Fig. 2 Additional blocks attached to the thighs. a Additional blocks with three thickness. The thicknesses of blocks were $10 \mathrm{~mm}, 20 \mathrm{~mm}$ and $30 \mathrm{~mm}$ from left, respectively. The blocks were rounded not to harm wearers. The unit of the ruler was millimeters. $\mathbf{b}$ A block attached to the right thigh with tapes. The tape passed around the thigh

was a little proximal knee joints position in order not to disturb knee flexion. The weights of the $10 \mathrm{~mm}$-thick block, $20 \mathrm{~mm}$-thick one and $30 \mathrm{~mm}$-thick one are $17 \mathrm{~g}$, $23 \mathrm{~g}$ and $29 \mathrm{~g}$, respectively. The weight of these blocks has little effects on running motions. The height and width of all blocks are $60 \mathrm{~mm}$ and $40 \mathrm{~mm}$, respectively. Block height and width were designed for avoiding its rotation on the thigh and attaching easily. The block edges are tapered to slide each other when they collide.

Each subject performed a trial in a day consisted of four bouts of 7-min running on a treadmill. Each bout was separated by at least 10-min resting interval, and each trial was separated by at least 1-day interval. Subjects performed four trials under each condition. The order of the conditions for a subject was randomly selected. Running speed in all bouts was $2.5 \mathrm{~m} / \mathrm{s}$ $(9 \mathrm{~km} / \mathrm{h})$. The subject ran without additional volume in the first bout (normal running). After this bout, the subject performed the other three bouts under selected condition out of four. The first bout was a control bout to obtain heart rates during normal running. The subjects were instructed to keep running speed constant and to run as usually as possible.

\section{Measurement systems}

Figure 3 shows axes and planes in the experiments. Running direction and the vertical axis were defined as $\mathrm{x}$-axis and y-axis, respectively.

A motion capture system (Motive, NaturalPoint, USA) was used to record the running motions. All cameras of the system (Flex 13, NaturalPoint, USA) captured 120 frames every second. A marker arrangement for the subjects was Rizzoli Lower Body Protocol [13-15], which is used in biomechanical study.

Motions of the subjects were calculated using inverse kinematics (IK) function of an open-sourced software for biomechanical analysis (OpenSim, The National Center for Simulation in Rehabilitation Research, USA). The IK function used a three-dimensional skeleton model with 23 degree of freedom (Gait2354Model). This skeleton model is standard for studies of gait and designed based on biomechanical studies [16-18]. The skeleton model was scaled to fit the subject bodies using marker positions of a static pose of the subjects and this static pose was T-pose. The IK function calculated the posture of the subjects, including joint angles, body parts' positions and positions of the center of mass $(\mathrm{CoM})$, based on marker trajectories during running. The solved CoM positions on at $\mathrm{x}$-axis and $\mathrm{z}$-axis were band-pass filtered $(1.0-6.0 \mathrm{~Hz})$ to remove noise and motions which periods were longer than stride time.

The subject wore a three-axes acceleration sensor on their right shoe to detect heel strikes. This sensor recoded acceleration at $142.86 \mathrm{~Hz}$ sampling frequency and its sensing range was $\pm 156.96 \mathrm{~m} / \mathrm{s}^{2}$ ( \pm 16 gravity acceleration). The acceleration signals were high-pass filtered $(60 \mathrm{~Hz})$ to remove signals during leg swinging. In the experiment, heel strikes detected as peaks of acceleration norm. Gait cycle ratio (GCR) represents phases of running motions. GCR of $0 \%$ means a heel strike of the right leg and GCR of $100 \%$ means next one. 


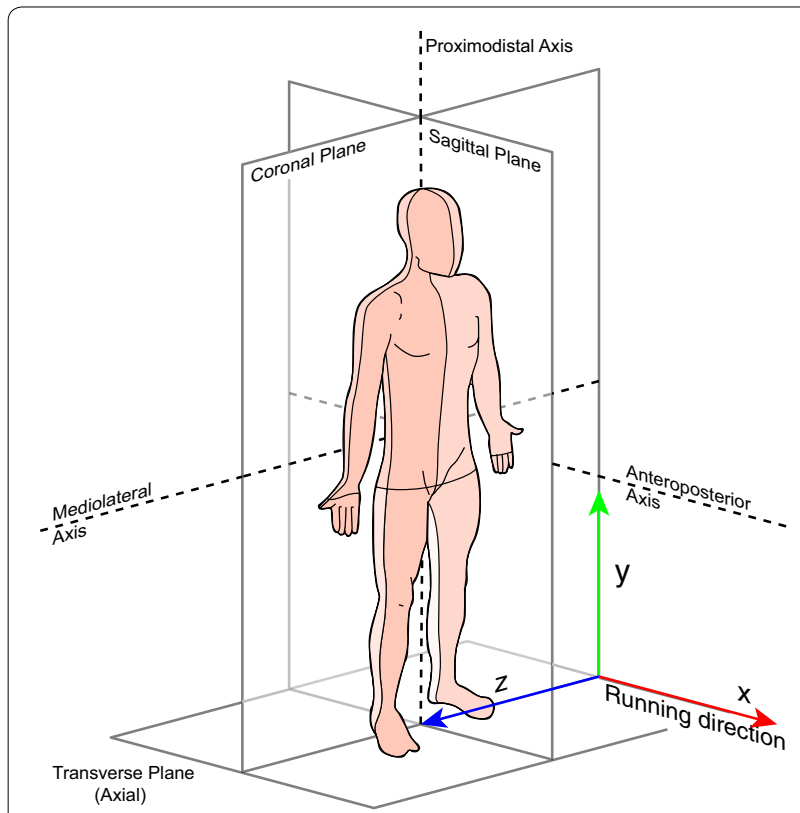

Fig. 3 Planes and axes around a runner and a running environment. In this paper, $x$-axis is a running direction of a treadmill and $x-z$ plane is upper face of the treadmill belt. An origin of the coordinate system is a left rear corner of the treadmill (This image is adapted from an open-sourced figure (https://upload.wikimedia.org/wikipedia/ commons/4/45/Anatomical_Planes-en.svg))

To obtain averaged signals during a single running cycle, signals were divided into one hundred parts according to GCR and averaged in each part. When we evaluated maximum and minimum values during a single running cycle, we calculated these from the divided values. To evaluate motions during a steady state, the values during 4-7 min were calculated.

A heart rates sensor using signals in an electrocardiogram (Polar H10, Polar Electro, Finland) was used to record metabolic demand of the subjects during the experiments. This sensor outputted data every $1 \mathrm{~s}$. The heart rates of the subject gradually increased from a beginning of running to approximately $3 \mathrm{~min}$ after the beginning and then became saturated. To evaluate the metabolic demand for steady running, the mean of measured heart rates during 4-7 min was used for evaluation.
To evaluate metabolic demand for running, we used net running heart rates which is calculated as heart rates minus the rates during the normal running on the same experimental day [19].

\section{Statistical analysis}

To evaluate difference in data among each condition, variables were statistically analyzed with the repeated measures analysis of variance (RM-ANOVA). In this paper, significance level, 0.05, was used for all statistical tests to determine significant difference. When RM-AROVA showed significant difference in groups, paired t-test evaluated difference in couples of groups.

Difference in each condition was sparsely analyzed at GCR of $0,25,50$ and $75 \%$. The instants at $0,25,50$ and $75 \%$ GCR represent characteristic events during running including, heel strike of right, the maximum knee flexion of right during the stance phase, the heel strike of left and the maximum knee flexion of left during the stance phase, respectively.

\section{Results}

Table 2 shows experimental data in each condition.

As shown in Table 2 and Fig. 4, the mean of the net running heart rates in the condition of $30 \mathrm{~mm}$-thick blocks was $6.05 \mathrm{bpm}$ higher than that in the condition of no

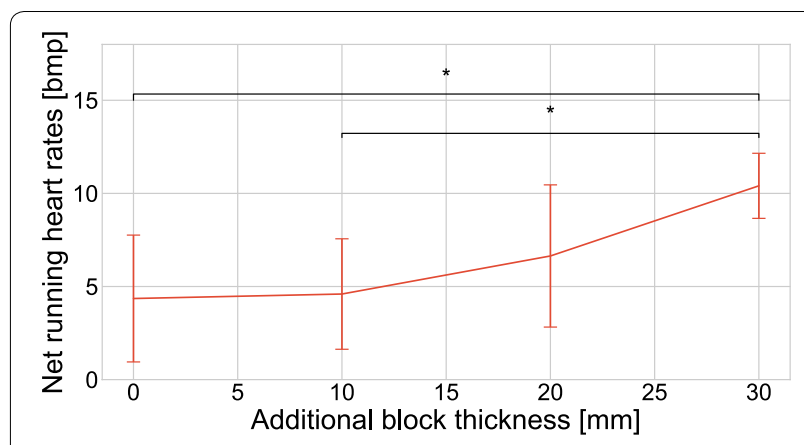

Fig. 4 Net running heart rates and additional block thickness. $30 \mathrm{~mm}$ thick blocks increased the heart rates compared to that without blocks. This single star $\left(^{*}\right)$ represents results of the paired t-test $(P<0.05)$. These error bars represented standard deviation of means dominated by inter-participant variability

Table 2 Statistical data of seven subjects running at speed of $2.5 \mathrm{~m} / \mathrm{s}$

\begin{tabular}{lrrrr}
\hline Variables & No block & 10 $\mathbf{~ m m ~ b l o c k ~}$ & 20 $\mathbf{~ m m ~ b l o c k ~}$ & $\mathbf{3 0 ~} \mathbf{~ m m ~ b l o c k}$ \\
\hline Net running heart rates (bpm) & $4.36 \pm 3.41$ & $4.6 \pm 2.97$ & $6.64 \pm 3.82$ & $10.41 \pm 1.75$ \\
Minimum knee distance (\%LL) & $18.06 \pm 1.22$ & $18.76 \pm 1.11$ & $20.09 \pm 1.88$ & $21.17 \pm 2.41$ \\
Minimum knee clearance (\%LL) & $4.20 \pm 1.79$ & $2.55 \pm 1.31$ & $1.53 \pm 2.17$ & $0.26 \pm 1.82$ \\
Step width (\%LL) & $5.63 \pm 2.51$ & $7.26 \pm 2.95$ & $8.32 \pm 3.31$ & $11.09 \pm 1.93$ \\
CoM amplitude on z-axis (\%LL) & $0.43 \pm 0.16$ & $0.43 \pm 0.14$ & $0.51 \pm 0.23$ & $0.66 \pm 0.28$ \\
\hline
\end{tabular}

The numbers are listed in format of mean $\pm \mathrm{sd}$ 
block $(P=0.023)$. The means of these in the conditions of $20 \mathrm{~mm}$-thick blocks and $10 \mathrm{~mm}$-thick blocks increased 2.28 and $0.24 \mathrm{bpm}$, respectively, which had no significant difference. $30 \mathrm{~mm}$-thick blocks increased physical load of running, but $20 \mathrm{~mm}$-thick blocks and $10 \mathrm{~mm}$-thick ones did slightly.

Figure 5 shows hip joint motions in the coronal plane during running. Positive and negative hip adduction angles represent leg adduction and abduction, respectively. The mean of the left hip adduction angle in the condition of $30 \mathrm{~mm}$-thick blocks was $3.50^{\circ}$ smaller than that with no block $(P=0.009)$. The mean of the angle in the condition of $20 \mathrm{~mm}$-thick blocks and $10 \mathrm{~mm}$-thick blocks increased by -3.80 and $0.03^{\circ}$ compared with the condition of no block, respectively, which had no significant difference. On the other hand, the mean of the right hip adduction angle had no statistically significance between all couples of the conditions. Hip adduction of the subjects was an asymmetric motion. An angle between the thighs in the coronal plane $\left(\phi_{t}\right)$ is defined as

$$
\phi_{t}=\phi_{r}+\phi_{l} \text {, }
$$

where $\phi_{r}$ and $\phi_{l}$ are the right hip adduction angle and left one, respectively. The $30 \mathrm{~mm}$-thick blocks reduced the mean of the thighs angle $\left(\phi_{t}\right)$ by $4.09^{\circ}$ compared with these without the blocks $(P=0.026)$. The means in the condition of $20 \mathrm{~mm}$-thick blocks and $10 \mathrm{~mm}$-thick blocks

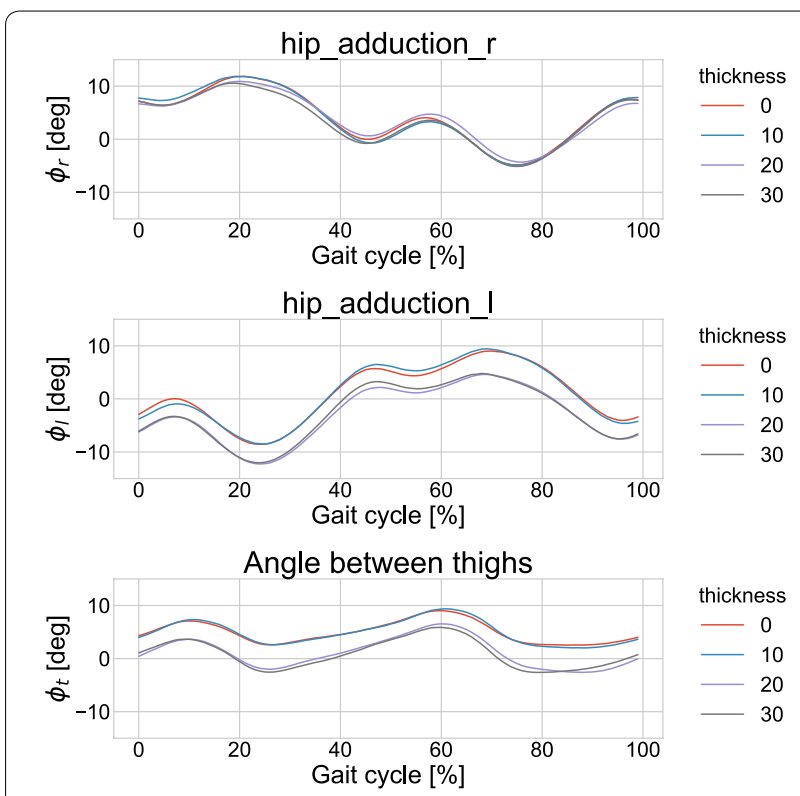

Fig. 5 Thigh motion at adducting/abducting direction during running. Positive $\phi_{r}$ and $\phi_{l,}$ and negative ones represent hip adduction and hip abduction, respectively. Positive and negative values of $\phi_{t}$ represent thighs opening and closing in the coronal plane, respectively. $0 \%$ and $100 \%$ gait cycle ratios represent the instants of right heel strikes decreased by $3.83^{\circ}$ and $0.03^{\circ}$ compared with the condition of no block, respectively, which had no significant difference. The subjects spread their legs during running with the $30 \mathrm{~mm}$-thick blocks.

The leg motions in the sagittal plane under each condition had little changes (Fig. 6). RM-ANOVA showed no statistically significant differences in the joint angles on the sagittal plane. The blocks in each thickness did not change leg motions in the sagittal plane.

Figure 7 shows relative positions of the knees to the pelvis during running. The $20 \mathrm{~mm}$-thick blocks and the $30 \mathrm{~mm}$-thick ones moved both knee trajectories in lateral direction. At $25 \% \mathrm{GCR}$, the $30 \mathrm{~mm}$-thick blocks decreased the mean of the left relative knee z-position by $1.94 \% \mathrm{LL}(P=0.007)$ compared with the condition of no block. At 75\% GCR, the $20 \mathrm{~mm}$-thick blocks and the $30 \mathrm{~mm}$-thick ones increased the means of the right knee relative z-position by 1.75 and $2.36 \% \mathrm{LL}$ $(P=0.008$ and 0.011$)$ compared with the condition of no block, respectively. The other differences of the right or left relative knee z-position between the condition of no block and the other conditions at the particular GCRs were not significant.

Figure 8 shows relationship between minimum distance between left and right knee positions $(d)$ and block thickness. The means of the minimum knee distance $(d)$ in the condition of $20 \mathrm{~mm}$-thick blocks and the $30 \mathrm{~mm}$ ones were $2.02 \% \mathrm{LL}$ and $3.11 \% \mathrm{LL}$ $(P=0.041$ and 0.021$)$ larger than the mean in the condition of no block, respectively. The mean in the condition of $10 \mathrm{~mm}$-thick blocks was $0.70 \% \mathrm{LL}$ higher than that in the condition of no block, and this difference was not significant.

Minimum knee clearance $(c)$ is defined as following equation,

$$
c=d-2(0.5 w-t)
$$

where $w$ and $t$ are knee width and block thickness, respectively. The minimum knee clearance $(c)$ represents a clearance between the additional blocks, however, actual block clearance is slightly larger than the minimum knee clearance because the block position is a little closer to the hip joints than knee position. Figure 9 shows relationship between the block thickness and the minimum knee clearance. When the additional blocks thickness increased, the minimum knee clearance decreased. The $10 \mathrm{~mm}, 20 \mathrm{~mm}$ and $30 \mathrm{~mm}$-thick blocks reduced the minimum knee clearance by $1.65,2.68$ and $3.94 \% \mathrm{LL}$ $(P=0.003,0.013$ and 0.006$)$ compared with that without the blocks, respectively. When the block thickness increased, a margin between the blocks for avoiding these collisions decreased. 

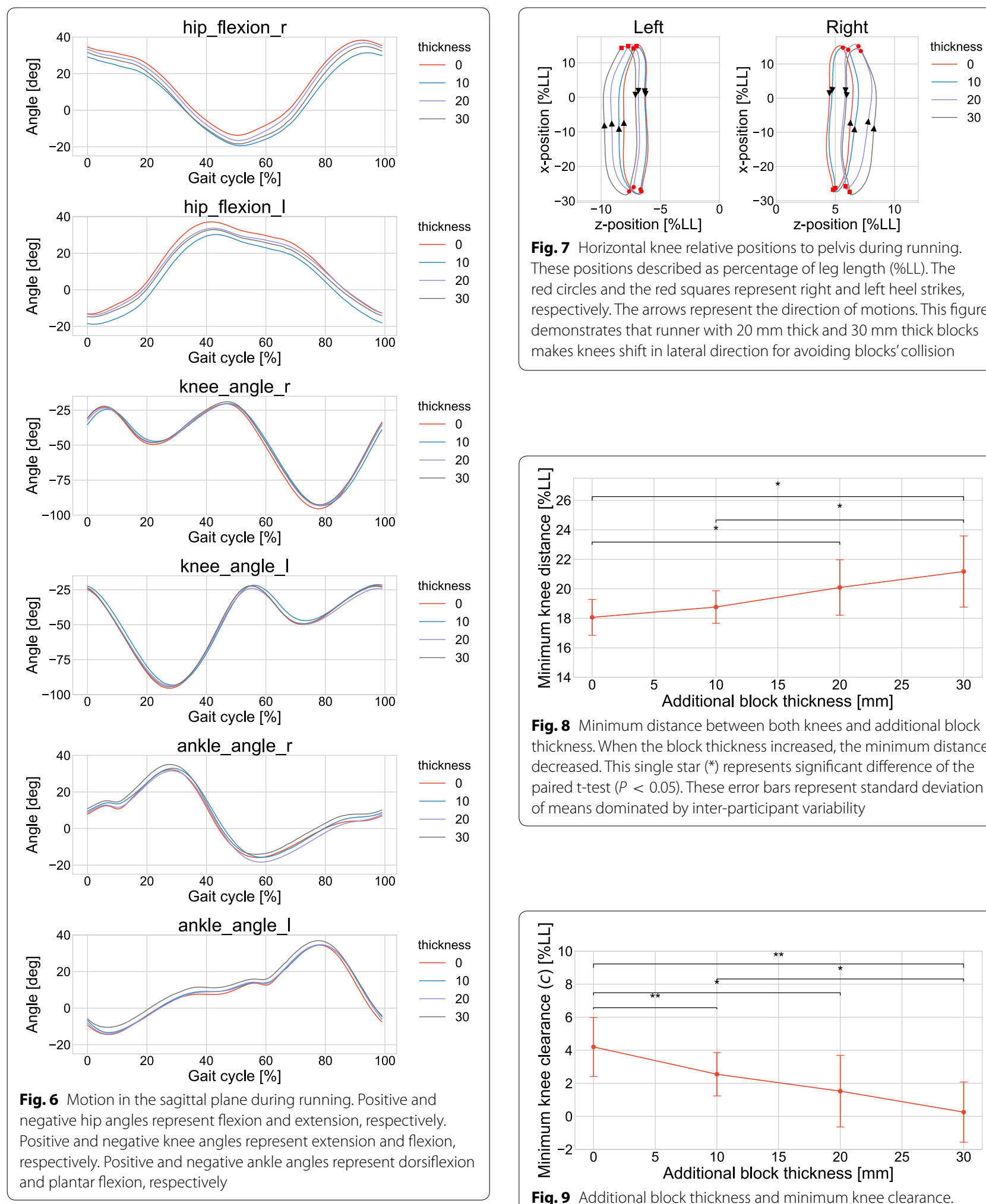

Fig. 7 Horizontal knee relative positions to pelvis during running. These positions described as percentage of leg length (\%LL). The red circles and the red squares represent right and left heel strikes, respectively. The arrows represent the direction of motions. This figure demonstrates that runner with $20 \mathrm{~mm}$ thick and $30 \mathrm{~mm}$ thick blocks makes knees shift in lateral direction for avoiding blocks' collision

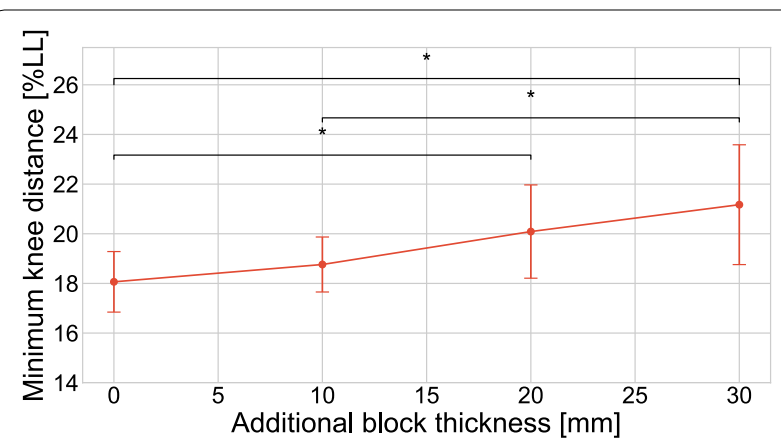

Fig. 8 Minimum distance between both knees and additional block thickness. When the block thickness increased, the minimum distance decreased. This single star $\left(^{*}\right)$ represents significant difference of the paired t-test $(P<0.05)$. These error bars represent standard deviation of means dominated by inter-participant variability

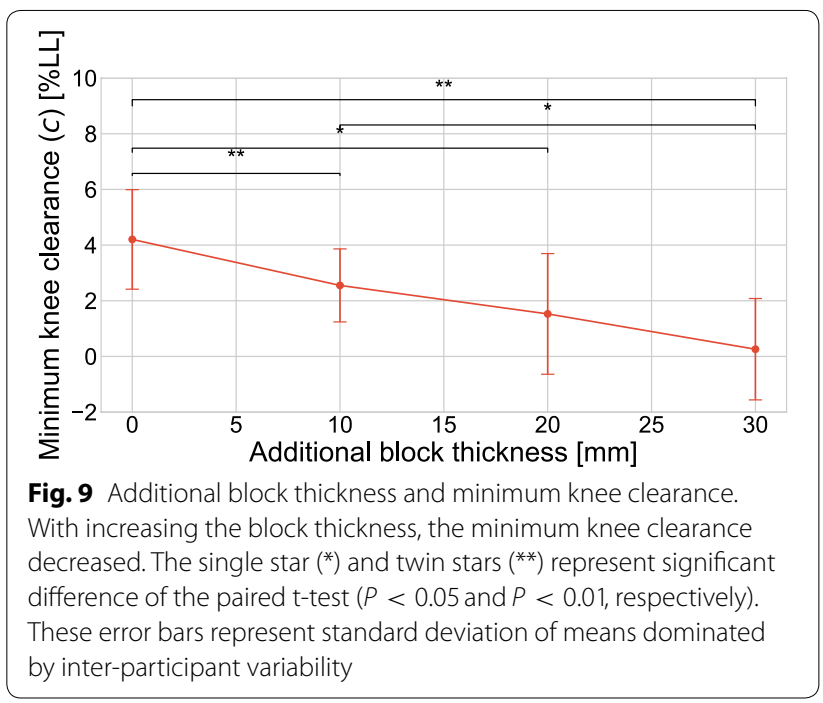


Figure 10 shows relative positions of the feet to the pelvis during running. Each origin of foot position is at a center of metacarpophalangeal joint of each index finger. The $20 \mathrm{~mm}$-thick blocks and the $30 \mathrm{~mm}$-thick ones moved right foot trajectory in lateral direction. Only $30 \mathrm{~mm}$-thick block also moved left foot trajectory in lateral direction. At $25 \%$ GCR, the $20 \mathrm{~mm}$-thick blocks and the $30 \mathrm{~mm}$-thick ones increased the means of right relative foot z-position by 1.47 and $2.92 \% \mathrm{LL}(P=0.006$ and 0.002) compared with the condition of no block, respectively. At $75 \%$ GCR, $30 \mathrm{~mm}$-thick blocks decrease the position of left by $2.53 \% \mathrm{LL}(P=0.041)$ compared with the condition of no block. The other differences of the right or left relative foot $\mathrm{z}$-position between the condition of no block and the other conditions at the particular GCRs were not significant.

Figure 11 shows relationship between step width and additional block thickness. The $10 \mathrm{~mm}, 20 \mathrm{~mm}$ and
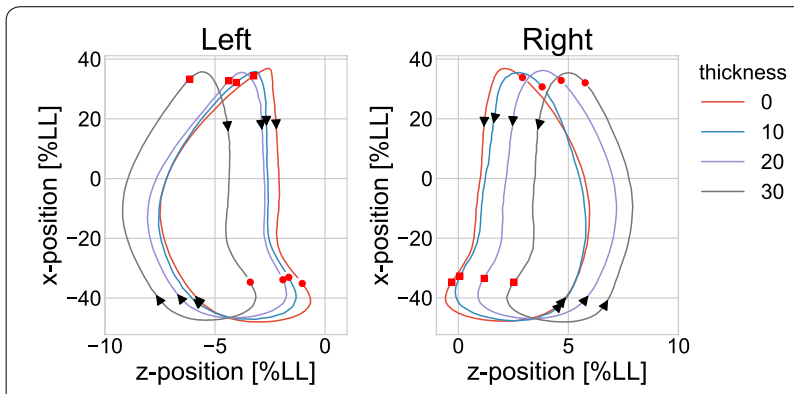

Fig. 10 Horizontal relative foot positions to pelvis during running. These positions were relative ones to the pelvis position. The red circles and the red square represent right and left heel strike, respectively. The arrows represent the direction of foot motions. This figure demonstrates that runner with $20 \mathrm{~mm}$ thick and $30 \mathrm{~mm}$ thick blocks moved foot in lateral direction

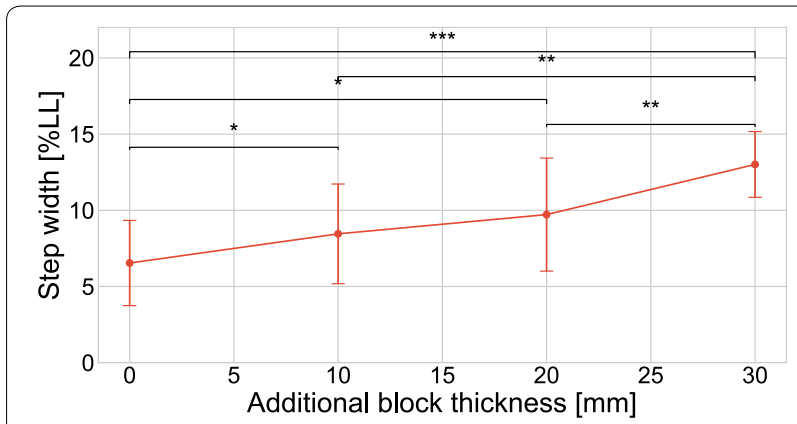

Fig. 11 Additional block thickness and step width. With increasing the block thickness, step width increased. Single stars $\left({ }^{*}\right)$, twin stars $\left.{ }^{(* *}\right)$ and triple stars $\left.{ }^{* * *}\right)$ represent results of the paired t-test $(P<0.05$ , $P<0.01$ and $P<0.001$, respectively). These error bars represented standard deviation of means dominated by inter-participant variability

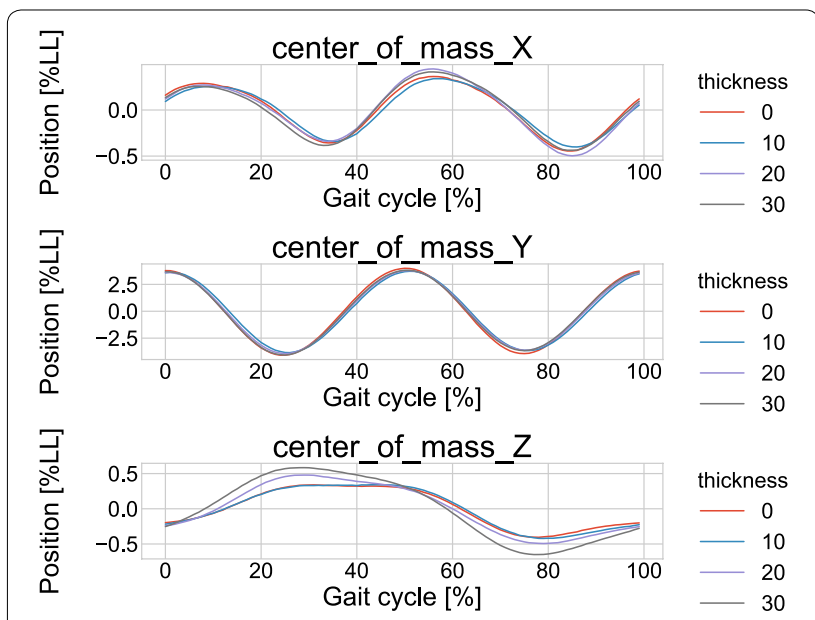

Fig. 12 The CoM position during running. The CoM position on $x$-axis and $z$-axis were band-pass filtered $(1.0-6.0 \mathrm{~Hz})$. The zero position on $x$-axis and $z$-axis does not represent the center of body, but means of position during running

$30 \mathrm{~mm}$-thick blocks increased the mean of step width by $1.91,3.18$ and $6.47 \% \mathrm{LL}(P=0.016, P=0.031$ and $P<0.001)$ compared with that without the blocks, respectively.

Figure 12 shows positions of the CoM during running. The CoM positions on $\mathrm{x}$-axis and $\mathrm{y}$-axis in each condition had little difference. The $30 \mathrm{~mm}$-thick blocks increased maximum z-positions of the CoM and decreased minimum ones. Figure 13 shows relationship between the block thickness and z-amplitude of the CoM during running. The $30 \mathrm{~mm}$-thick blocks increased the mean of the amplitude by $0.27 \% \mathrm{LL}(P=0.045)$ compared with that without the blocks. The CoM during running with the $30 \mathrm{~mm}$-thick blocks was strongly accelerated on the mediolateral axis.

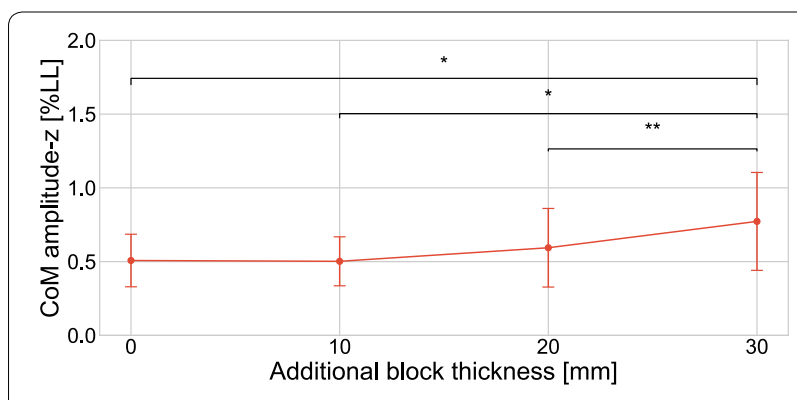

Fig. 13 Amplitude of the CoM motion on z-axis and additional block thickness. $20 \mathrm{~mm}$ and $30 \mathrm{~mm}$ thick blocks increased the amplitude. The single star $\left(^{*}\right)$ and twin stars $\left(^{* *}\right.$ ) represent significant difference of the paired t-test $(P<0.05$ and $P<0.01$, respectively). These error bars represent standard deviation of means dominated by inter-participant variability 


\section{Discussion}

The joint trajectories and the CoM motion during normal running were reported $[20,21]$. The results of the trajectories of normal running in our experiments and these conventional research results were consistent in joint angles and CoM motions.

The blocks on medial side of the thighs can reduce margin between the thighs. To keep running speed constant, runners spread their legs to compensate the margin and to avoid collision of the blocks. There were few collisions between the blocks happened during the experiments, since the subjects had known the size of the attached blocks before running. They unconsciously changed their running motions for the collision avoidance in advance. The thighs angle $\left(\phi_{t}\right)$ in the conditions of $20 \mathrm{~mm}$-thick blocks and $30 \mathrm{~mm}$-thick ones decreased by $77 \%$ and $82 \%$ compared with that without blocks, respectively (Fig. 5). This reduction supports the hypothesis that a runner spreads their legs to avoid collision of the blocks.

When the thighs angle decreased, the relative knee joint positions shifted in lateral direction, and minimum knee distance increased to obtain an enough clearance between the blocks for avoiding these collisions. As shown in Fig. 7, the relative knee joint positions during running with the $20 \mathrm{~mm}$ and $30 \mathrm{~mm}$-thick blocks moved in lateral direction. When the pelvis rotates, the relative knee joint position moves even if right hip adduction is constant. The means of the pelvis rotation in the condition of $30 \mathrm{~mm}$ blocks and $20 \mathrm{~mm}$ blocks decreased by $2.71^{\circ}$ and $3.04^{\circ}$ compared with the condition of no block, respectively. As a result, the right knee relative position moved in the lateral direction.

Trajectories of the knee positions kept these profiles constant in each case. Runners with the blocks do not decrease hip adduction angle in a limited time around the instance when the knees passed each other, because changing the profiles can increase metabolic demand for running. The minimum knee clearance $(c)$ decreased when the thickness of blocks attached to the knees increased. We assume that runners keep the minimum clearance constant by increasing hip abduction angles. However, subjects in these experiments did not actually increase their hip abduction angles. Changing knee joint trajectories from these of normal running increases required human effort. Runners wearing the blocks need both to keep the minimum knee clearance positive and to suppress changing the trajectories. When the block thickness increased in the experiments, the latter's priority increased.

The hip abduction shifts foot positions in lateral direction and increases the step width. $20 \mathrm{~mm}$-thick blocks moved right foot position in lateral direction, and
$30 \mathrm{~mm}$-thick blocks moved both foot positions in lateral direction (Fig. 10). The runners keep horizontal profiles of the foot position constant as well as that of knee.

Amplitude of the CoM position on the mediolateral axis with $30 \mathrm{~mm}$-thick blocks was larger than that without blocks. The amplitude with the $10 \mathrm{~mm}$ and $20 \mathrm{~mm}$-thick blocks had no significant difference from that without blocks. Small increase in step width does not increase the amplitude because the runners can keep their pelvis position coincided with that of normal running.

Right knee and foot positions were closer to pelvis positions than left ones. This result shows that right leg was more vertical to the ground in the coronal plane than left ones, and that the CoM position was biased to right. The all subjects' handed-legs was right, and they mainly used their handed-leg for acceleration of the CoM.

All the net running heart rates in this experiment were higher than 0 . Heart rates during running under experimental conditions were higher than that during the first bout. Fatigue of a runner during the first bout increased those. $30 \mathrm{~mm}$ blocks increased net running heart rates by $6.05 \mathrm{bpm}$ compared with that without blocks, but the other blocks did not significantly.

Arellano et al. [12] reported U-shaped relationship between net metabolic power and step width. The metabolic power during running assumes to be minimum when a runner takes preferable step width. With increased and decreased step width from preferred one, the metabolic power increases. Change rates of metabolic power increase with increase of step width from preferred one. In our experiment, the additional blocks increased step width of runners. Small increased step widths brought by the $10 \mathrm{~mm}$-thick blocks and the $20 \mathrm{~mm}$-thick ones had less effects on the net running heart rates, because the step widths were close to preferred ones. On the other hand, the $30 \mathrm{~mm}$-thick blocks increased the heart rates, because these step width was far from preferred ones. We assume that the heart rates and the block thickness have also an U-shaped relationship with step width that the heart rates increase sharply between the thickness of $20 \mathrm{~mm}$ and $30 \mathrm{~mm}$. Arellano et al. explained that un-preferred step width increases ground reaction force (GRF) in the mediolateral direction and the CoM moment around the anteroposterior axis. GRF in the mediolateral direction increases the CoM amplitude in the mediolateral direction and disturbs effective progress of a runner. When the moment around the anteroposterior axis increases with increase of step width, runners need counter moment to keep their torso orientation upright. Runners with un-preferred step width need high effort for moving forward and keeping the torso orientation. The $30 \mathrm{~mm}$-thick blocks attached 
to medial sides of the thighs increase CoM amplitude in the mediolateral direction and increased the heart rates.

\section{Conclusion}

In summary, our data reveals that $30 \mathrm{~mm}$-thick blocks attached to medial sides of the thighs increased net running heart rates by $6.05 \mathrm{bpm}$, and amplitude of the CoM on the mediolateral axis [\%LL] by $52 \%$ compared to these in normal running. On the other hand, the $10 \mathrm{~mm}$-thick blocks and the $20 \mathrm{~mm}$-thick ones did not increase neither net running heart rates and the CoM amplitude. This result shows that running can be disturbed by medial parts of a running assistive device which thickness is more than $30 \mathrm{~mm}$ around medial side of the knee joint. To avoid adverse effects on running, thickness of medial parts of the device should be less than $30 \mathrm{~mm}$.

\section{Acknowledgements}

This work was supported by JSPS KAKENHI Grant Number JP17H03205.

\section{Authors' contributions}

NK designed the experiment, carried out all experiments, analyzed data and wrote the paper. YH initiated this project and gave advises on the design of the experiment, analysis of data and paper writing. Both authors read and approved the final manuscript.

\section{Competing interests}

The authors declare that they have no competing interests.

\section{Author details}

${ }^{1}$ The Department of Micro-Nano Systems Engineering, Nagoya University, Furo-cho 1, Chikusa-ku, Nagoya, Aichi 464-8603, Japan. ${ }^{2}$ The Department of Micro-Nano Mechanical Science and Engineering, Nagoya University, Furo-cho 1, Chikusa-ku, Nagoya, Aichi 464-8603, Japan.

Received: 6 February 2019 Accepted: 1 July 2019

Published online: 09 July 2019

\section{References}

1. Herr H (2009) Exoskeletons and orthoses: classification, design challenges and future directions. J Neuroeng Rehabil 6(1):21. https://doi. org/10.1186/1743-0003-6-21

2. Grabowski AM, Herr HM (2009) Leg exoskeleton reduces the metabolic cost of human hopping. J Appl Physiol 107(3):670-678. https://doi. org/10.1152/japplphysiol.91609.2008. http://jap.physiology.org/conte nt/107/3/670.full.pdf

3. Kitatani R, Ohata K, Takahashi H, Shibuta S, Hashiguchi Y, Yamakami N (2014) Reduction in energy expenditure during walking using an automated stride assistance device in healthy young adults. Arch Phys Med Rehabil 95(11):2128-2133. https://doi.org/10.1016/j.apmr.2014.07.008

4. Collins SH, Wiggin MB, Sawicki GS (2015) Reducing the energy cost of human walking using an unpowered exoskeleton. Nature 522(7555):212-215
5. Mooney LM, Rouse EJ, Herr HM (2014) Autonomous exoskeleton reduces metabolic cost of human walking during load carriage. J Neuroeng Rehabil 11(1):80. https://doi.org/10.1186/1743-0003-11-80

6. Mooney LM, Herr HM (2016) Biomechanical walking mechanisms underlying the metabolic reduction caused by an autonomous exoskeleton. J Neuroeng Rehabil 13(1):4. https://doi.org/10.1186/s12984-016-0111-3

7. Lee G, Kim J, Panizzolo FA, Zhou YM, Baker LM, Galiana I, Malcolm P, Walsh CJ (2017) Reducing the metabolic cost of running with a tethered soft exosuit. Sci Robot 2:eaan6708

8. Kim J, Heimgartner R, Lee G, Karavas N, Perry D, Ryan D, Eckert-Erdheim A, Murphy P, Choe D, Galiana I, Walsh C (2018) Autonomous and portable soft exosuit for hip extension assistance with online walking and running detection algorithm. In: IEEE international conference on robotics and automation (ICRA), Brisbane, Australia, May 21-25

9. Bourdin M, Belli A, Arsac LM, Bosco C, Lacour JR (1995) Effect of vertical loading on energy cost and kinematics of running in trained male subjects. J Appl Physiol 79(6):2078-2085. https://doi.org/10.1152/jappl .1995.79.6.2078

10. Martin P (1985) Mechanical and physiological responses to lower extremity loading during running. Med Sci Sports Exerc 17(4):427-433

11. Donelan JM, Kram R, Kuo AD (2001) Mechanical and metabolic determinants of the preferred step width in human walking. Proc R Soc Lond 268(1480):1985-1992. https://doi.org/10.1098/rspb.2001.1761

12. Arellano CJ, Kram R (2011) The effects of step width and arm swing on energetic cost and lateral balance during running. J Biomech 44(7):12911295. https://doi.org/10.1016/j.jbiomech.2011.01.002

13. Leardini A, Sawacha Z, Paolini G, Ingrosso S, Nativo R, Benedetti MG (2007) A new anatomically based protocol for gait analysis in children. Gait Posture 26(4):560-571. https://doi.org/10.1016/j.gaitpost.2006.12.018

14. Leardini A, Biagi F, Merlo A, Belvedere C, Benedetti MG (2011) Multisegment trunk kinematics during locomotion and elementary exercises. Clin Biomech 26(6):562-571. https://doi.org/10.1016/j.clinbiomec h.2011.01.015

15. Leardini A, Benedetti MG, Berti L, Bettinelli D, Nativo R, Giannini S (2007) Rear-foot, mid-foot and fore-foot motion during the stance phase of gait. Gait Posture 25(3):453-462. https://doi.org/10.1016/j.gaitpost.2006.05.017

16. Anderson FC, Pandy MG (1999) A dynamic optimization solution for vertical jumping in three dimensions. Comput Methods Biomech Biomed Eng 2(3):201-231. https://doi.org/10.1080/10255849908907988

17. Yamaguchi GT, Zajac FE (1989) A planar model of the knee joint to characterize the knee extensor mechanism. J Biomech 22(1):1-10. https://doi. org/10.1016/0021-9290(89)90179-6

18. Delp SL, Loan JP, Hoy MG, Zajac FE, Topp EL, Rosen JM (1990) An interactive graphics-based model of the lower extremity to study orthopaedic surgical procedures. IEEE Trans Biomed Eng 37(8):757-767. https://doi. org/10.1109/10.102791

19. Schrack JA, Zipunnikov V, Goldsmith J, Bandeen-Roche K, Crainiceanu CM, Ferrucci L (2014) Estimating energy expenditure from heart rate in older adults: a case for calibration. PLOS ONE 9(4):1-9. https://doi. org/10.1371/journal.pone.0093520

20. Novacheck TF (1998) The biomechanics of running. Gait Posture 7(1):77-95. https://doi.org/10.1016/S0966-6362(97)00038-6

21. Preece SJ, Mason D, Bramah C (2016) The coordinated movement of the spine and pelvis during running. Hum Mov Sci 45:110-118. https://doi. org/10.1016/j.humov.2015.11.014

\section{Publisher's Note}

Springer Nature remains neutral with regard to jurisdictional claims in published maps and institutional affiliations. 